\title{
Magnetic determination of the Current Center Line for the superconducting ITER TF coils
}

\author{
Philippe Lerch, Karl Buhagiar, Daniel Wassmer, Alexander Gabard, Alberto Barutti, Marco Buzio, \\ Nicholas Sammut, Stéphane Sanfilippo, Arnaud Foussat
}

\begin{abstract}
The ITER tokamak includes 18 superconducting D-shaped toroidal field (TF) coils. Unavoidable shape deformations as well as assembly errors will lead to error fields in the final configuration, which can be modeled with the knowledge of the current center line (CCL). We are building a room temperature magnetic measurement system using low frequency $a c$ excitation current through the TF coil and arrays of pick-up coils, fabricated with printed circuit board technology. Deviations from the expected shape of the CCL will be obtained by comparing the amplitude of magnetic flux measured at several locations around the perimeter of the TF coil, with values computed assuming the nominal current distribution. We present experimental results obtained with a cable placed in one turn groove of a full scale radial plate.
\end{abstract}

Index Terms-ITER, TF coils, fluxmeter, inverse problem

\section{INTRODUCTION}

D EPARTURE from the ideal magnetic field axisymmetry in a Tokamak is mainly due to deviations from the perfect magnetic field configuration [1]. These deviations cause error fields and are the result of manufacturing and installation tolerances, the presence of joints and busbars, as well as the presence of ferromagnetic elements [1]. The current center line (CCL) of a magnet is defined as the 3D barycenter filament computed with the spatial distribution of current (i.e cables) of the assembly. True shape deformations of this filament are used to model error fields. For ITER magnets [2], the reference CCL of the toroidal field (TF) coils is defined [3]. Several contributions modeling such a measurement[4], [5], [6], [7], describing the validation of the measurement procedures [8], [9] as well as discussing manufacturing [10], [11] and assembly [12], [13] issues are available.

We are developing a prototype measurement system capable to detect deviations from the nominal shape of the CCL with a precision ranging between 1 and $3 \mathrm{~mm}$. In the forward approach, a known current filament allows to compute the

Manuscript received August 13, 2014. Contact: philippe.lerch@psi.ch This work is supported in part by ITER Organization D 4HBLS3 $\mathrm{Ph}$. Lerch is with Paul Scherrer Institute, CH-5232 Villigen, Switzerland K. Buhagiar is with University of Malta, MSD2080-Msida Malta

D. Wassmer is with Paul Scherrer Institute, CH-5232 Villigen, Switzerland A. Gabard is with Paul Scherrer Institute, $\mathrm{CH}-5232$ Villigen, Switzerland

A. Barutti is with ASG Superconductors S.p.A. 16152-Genova, Italy

M. Buzio is with CERN, CH-1203 Geneva, Switzerland

N. Sammut is with University of Malta, MSD2080-Msida, Malta

A. Foussat is with ITER, 13108-Saint-Paul-Lez-Durance, France expected magnetic field density maps (or gradients thereof) and to compare the result to experimental data. Eventually, the system must be capable to quantify filament shape deviations using precision magnetic survey and nominal shape data as input, i.e. provide the solution of an inverse problem. This contribution addresses practical issues of measuring known filament shapes in the forward approach and presents preliminary results obtained with an inversion algorithm.

\section{EXPERIMENT}

\section{A. Context}

The proof of principle was obtained by introducing known deformations in a 1:5 scale single filament laboratory model [14], and detecting the corresponding magnetic signal differences. The timeline of this project supported by IO collides with the manufacturing schedule of the coil elements, which are required to validate the prototype under development. Therefore, any alternative delivering real scale information, in particular on the negative effects of eddy currents flowing in the steel structure and acting against the main field, is a valuable asset. A time window was identified to perform, on the premises of the industrial partner ASG - La Spezia, Italy, a "simplified CCL" experiment using the first available radial plate, in which a one turn cable was installed.

\section{B. Principle}

We briefly recall the spirit of the detection method [15]. A low frequency $\omega / 2 \pi$ excitation current $I=I_{0} \cdot \cos (\omega t)$ flows through the coil to be measured. Induced voltage results from the time variation of magnetic flux $\phi$. Thus, $\dot{\phi}=\delta \Phi / \delta t \propto \omega \cdot \phi$ values are recorded with arrays of calibrated pick-up coils manufactured with printed circuit board (PCB) technology. Each array accommodates 8 sensors. Two arrays are mounted on adjacent faces of a cubic shape support and sense two orthogonal components of magnetic flux, one along the vertical $\mathrm{z}$-axis and a radial component lying in the $\mathrm{XY}$ plane. The array oriented to measure $\dot{\phi}_{z}$ samples magnetic flux along the radial direction, $\dot{\phi}_{z}=\dot{\phi}_{z}(r)$, whereas the orthogonal device probes along the $z$-axis, $\dot{\phi}_{r}=\dot{\phi}_{r}(z)$, see inset of Fig. 2.

An optical laser tracker points at three targets mounted on the detector cube and records its location, $P_{\text {cube }}$, in space. A separate calibration links $P_{\text {cube }}$ to the centers of the 16 flux coils $P_{k}(1, \ldots, 16)$. A survey around the perimeter of a Dshape magnet produces two maps of raw data $V_{z, e}\left(P_{k}(r), \omega\right)$ and $V_{r, e}\left(P_{k}(z), \omega\right)$. The second subscripts $e$ and $m$ are for experimental and model values, respectively. 
The precise knowledge of the geometry of the current filament $\operatorname{CCL}\left(x_{i}, y_{i}, z_{i}\right)$, and $P_{k}$ allows us to compute the field maps $B_{z, m}\left(P_{k}(r)\right)$ and $B_{r, m}\left(P_{k}(z)\right)$ using the Biot and Savart relation. Eddy currents are excluded but their impact will be discussed below. For each pick-up coil in the arrays, the largest of 14 spirals is $410 \mathrm{~mm}$ x $36 \mathrm{~mm}$ in size, and centered around $P_{k}$; the PCB has 22 layers, connected in series. The actual quantity of interest is the relative difference between the measured and computed magnetic flux. By introducing the details of the coils, we compute the corresponding flux maps $\phi_{z, m}\left(P_{k}(r), \omega\right)$ and $\phi_{r, m}\left(P_{k}(z), \omega\right)$ for every measured location $P_{k}$. Best agreement with data is obtained by adjusting the scaling factor $S F$ and allowing for minute coordinate systems adjustments. Further improvement (i.e. smaller differences) can be obtained by including some educated guess describing a variation of the shape of the filament. The solution of the inverse problem (invokes a first order development, see [15]) requires the computation of the pseudo-inverse matrix of the Jacobian describing $B$ [16], [20]. A realistic set of degrees of freedom that capture shape deviations commensurate with the specifications of the assembly, the stiffness and sizes of the materials, is required. In addition to noise, factors unrelated to the winding geometry but truly capable of modifying the magnetic flux density profiles like reinforcement iron in the concrete floor of the hall, eddy currents, as well as electrical contacts to the cable are likely sources of concern.

\section{FORWARD APPROACH: WITH A 1:1 RADIAL PLATE}

\section{A. Preparation}

In this experiment, a simplified CCL was wound in one full turn groove of a radial plate maintained horizontally on wood supports, in the premises of ASG - La Spezia. The concrete of the floor is free from magnetic material. A commercial $27 \mathrm{~mm}$ diameter power cable including five $16 \mathrm{~mm}^{2} \mathrm{Cu}$ conductors embedded in insulating material was placed inside the groove; the conductors are connected in series. The cable is maintained every $\approx 700 \mathrm{~mm}$ in the groove by dedicated wooden jigs and kept in place inside the groove by plastic wedges. The jigs were manufactured to take advantage of the flat portion of the groove sides. Fig. 1 illustrates the arrangement. Between the jigs, the cable rests in the groove by gravity. The cable is connected to three ac amplifiers (Kepco $20 \mathrm{~V}$ - 20A) connected in parallel in a master-slave configuration. The maximum current was $60 \mathrm{Amp}$ (5 . $60 \mathrm{Amp} \cdot$ turns) and the excitation frequency was varied between 1 and $9 \mathrm{~Hz}$. The master amplifier was driven by a signal generator.

Using another purposely designed jig, 135 survey points of the position of the cable were recorded with a laser tracker. Interpolation is performed on this data to obtain a discrete set of coordinates describing the current filament $\mathrm{CCL} \mathrm{T}_{e}$.

\section{B. Experiment}

The acquisition of the 16 values involves the use of two 8 channels adc cards [17] and ad-hoc acquisition code (Labview). For each channel, a pick-up coil of $\approx 4.5 \mathrm{~m}^{2}$ flux collection area [15] is connected in series with a passive RC filter, and then to the acquisition card. Digitized waveforms,

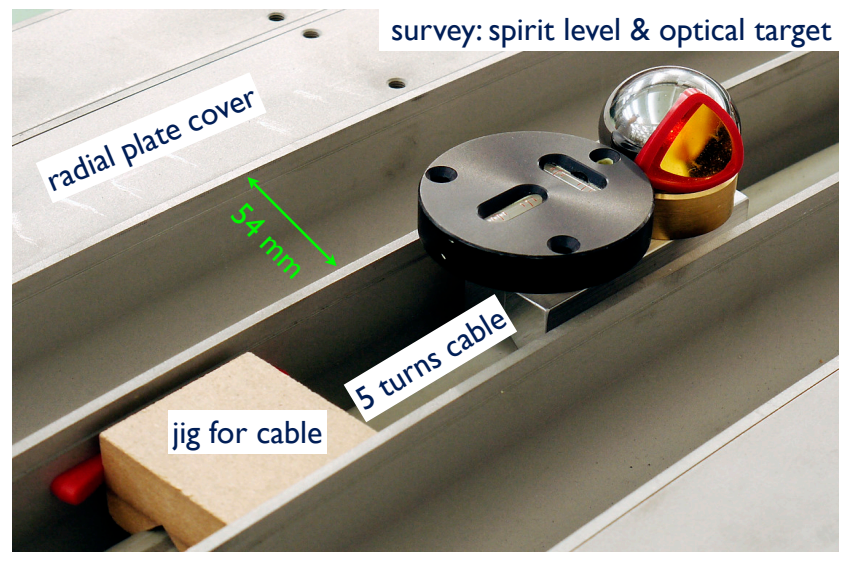

Fig. 1. Details of one wood jig used to maintain the cable in one of the grooves and the tools used to survey the conductor around the radial plate.

adjusted to record 8 full excitation cycles, are best-fit to a sine wave function and deliver the voltages $V_{r, e}\left(P_{k}, z\right)$ and $V_{z, e}\left(P_{k}, r\right)$ induced by the flux variation. The frequency response of each $\mathrm{RC}$ filter as well as minute gain differences between the channels are corrected in dedicated calibration steps. At each location $P_{k}$, (uncertainty 100 micrometers) the average of 30 acquisition cycles is recorded; electronic noise at $1 \mathrm{~Hz}$ is $\approx 10$ microvolts.

\section{Results}

This experiment delivers, for each component, 8 coils $\mathrm{x} 52$ locations, (i.e. $k$ of $P(k)$ ranges from 1 to 416 ) experimental values. Combining with survey data, maps of induced voltage $V_{r, e}\left(P_{k}(z)\right)$ and $V_{z, e}\left(P_{k}(r)\right)$ are assembled. Using the survey data $\mathrm{CCL} \mathrm{T}_{e}$ we compute $B_{m, z}\left(P_{k}(r)\right)$ and $B_{m, r}\left(P_{k}(z)\right)$ at each location $P_{k}$, which is the center of the actual flux collection area of the pick-up coil. Since the magnetic flux density across a coil is not constant, we segment the coil area in $2 \times 4$ sub regions. The theoretical flux density $\phi$ in the center of each sub-region is approximated by the average flux density obtained with the values computed at each corner. The collected flux is obtained by summation. We recall that $V_{i}=\delta \phi_{i} / \delta t \propto \omega \cdot B_{i}$ and compute the maps of the relative differences for the $i=r$, radial and $i=z$, z-axis components

$$
\delta V_{i}=\frac{V_{i, e}\left(P_{k}\right)-S F \cdot V_{i, m}\left(P_{k}\right)}{V_{i, e}\left(P_{k}\right)} .
$$

Fig. 2 shows the contour plot of the relative induced voltage differences $\delta V_{z}\left(P_{k}(r)\right)$ expressed in the $\mathrm{XY}$ frame of the radial plate. The data describing $\mathrm{CCL} \mathrm{T}_{e}$, and the $(52 \times 8)$ coordinates of the $P_{k}$ are shown as well. As anticipated, the contour lines do reflect the global shape of the current source.

The RP is not a flat and stiff object. The cable has twists and turns and the survey with only 135 points likely misses a few of these. However, the Z-variation of the cable follows the actual shape of the RP accurately. The relative difference, $\epsilon\left(\delta V_{i}\right)_{r m s}$ at $1 \mathrm{~Hz}$ are $0.025(2)$ and $0.040(9)$ for the zaxis and r-components, respectively. This result includes the contribution from two regions; $\mathrm{XY}=(2000 \mathrm{~mm},-6500 \mathrm{~mm})$ 
This is the author's version of an article that has been published in this journal. Changes were made to this version by the publisher prior to publication. The final version of record is available at http://dx.doi.org/10.1109/TASC.2014.2360986

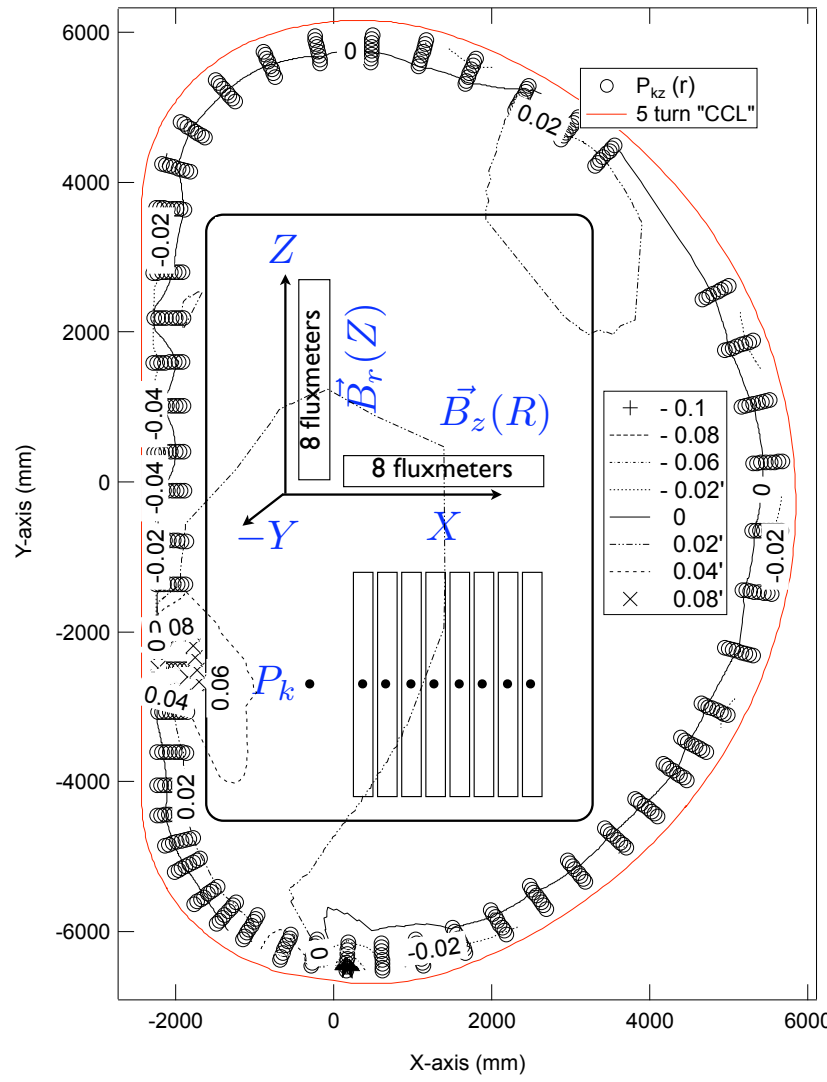

Fig. 2. Contour plot of the relative z-axis field component difference at 1 $\mathrm{Hz}$ measured at $P_{k}$ and computed using CCL5T $e$ current filament data. The insets illustrate the arrangement of the sensor arrays.

$\delta V_{z}$ and $\mathrm{XY}=(0,-2500 \mathrm{~mm})$ where series connection and contact to the power supply take place. If one ignores these two regions (5 locations, i.e. $5 \times 8 P_{k}$ values), $\epsilon\left(\delta V_{i}\right)_{r m s}$ reduces to $0.013(1)$ and $0.017(6)$ for the $\mathrm{z}$-axis and radial components, respectively.

\section{FREQUENCY DEPENDENCE}

Eddy currents flowing in the metallic structure of the RP are of concern, for they induce reaction fields acting against the forward field produced by the source. In order to quantify this effect, we recorded data at 4 different frequencies, compute the relative differences $\delta V_{z}\left(P_{k}(r)\right)$ and $\delta V_{r}\left(P_{k}(z)\right)$ for three frequencies and show the result in Fig. 3 as a function of $P_{k}$. The first 52 points represent data recorded by coil 1 of the PCB arrays whereas the last 52 points are data from coil 8. Data at $1 \mathrm{~Hz}$ is the same as the one shown in Fig. 2, but locations near electrical contact regions are ignored. As already anticipated, the best agreement between measured and expected induced voltage (i.e. the smallest $\delta V_{i}\left(P_{k}\right)$ ) is obtained at $1 \mathrm{~Hz}$ for both field components. As frequency increases, $\delta V_{i}\left(P_{k}\right)$ exhibits an increasing negative offset due to the loss of signal caused by eddy currents flowing in the metallic structure. More important though is the observation that the $\mathrm{z}$-axis component $\delta V_{z}\left(P_{k}(r)\right)$ depends on the position of the pick-up coil with

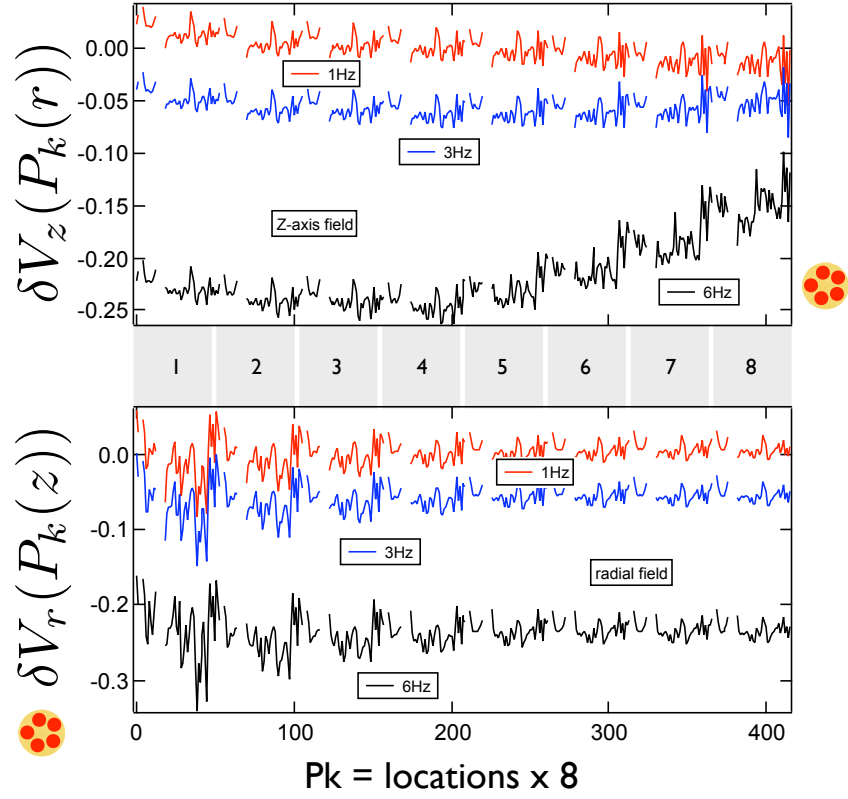

Fig. 3. Relative difference $\delta V_{i}\left(P_{k}\right)$ between measured and computed signal for different frequencies. The source cable (symbol) is closer to coil 1 for the radial component.

respect to the field source. The pronounced knee observed at 6 $\mathrm{Hz}$ around coil 4 in $\delta V_{z}\left(P_{k}(r)\right)$ enhances further at $9 \mathrm{~Hz}$ (not shown). We are currently investigating [18] this behavior. Even at $1 \mathrm{~Hz}$, the small negative slope visible in the $\delta V_{z}\left(P_{k}(r)\right)$ data indicates that eddy currents cannot entirely be neglected.

\section{SEnsitivity}

Our model neglects signal loss due to eddy current. It is nevertheless possible to use the $1 \mathrm{~Hz}$ data to estimate the available detection sensitivity. Table 1 shows the relative agreement, expressed by the rms value $\epsilon_{i}$ of $\delta V_{i}\left(P_{k}\right)$; perfect match would yield $\epsilon=0$. The scaling factor $S F$ recovers imprecision in the amplitude of the excitation current. Sensitivity is quantified with the $z$ axis variations of $\operatorname{CCL5T}(z)$, which accurately follow the shape of the radial plate groove edge. We re-compute $\delta V_{i, m}\left(P_{k}, \operatorname{CCL} \mathrm{T}_{e}(\mathrm{x}, \mathrm{y}, \bar{z})\right)$ and observe a clear degradation of both field components; the global agreement factors $\epsilon_{i}$ increase.

In order to validate this observation, we compare the signal amplitude associated to the filament height modulation $(z-\bar{z})$ to the propagated errors (quadratic sum) contained in the experiment. For $P_{k}$, survey uncertainties for each degrees of freedom and $P_{\text {cube }}$ to $P_{k}$ calibration contributes with $\approx 10$ $\mu \mathrm{V}$ to the uncertainty, noise at $1 \mathrm{~Hz}$ is $\approx 10 \mu \mathrm{V}$, so that the propagated uncertainty is estimated to be $40 \mu \mathrm{V}$. The relative error in the flux calculation across the coil's area $\sigma_{c} \approx 3-5 \cdot 10^{-4}$ as well as the effect due to the variation of one attitude angle of the detector assembly, are ignored. With these assumptions, the signal $\left(V_{r}\left(P_{k}(z)\right)-V_{r}\left(P_{k}(\bar{z})\right)\right)$ associated to the height modulation of CCL5T is computed along the $34 \mathrm{~m}$ filament length and shown in Fig. 4, together with CCL5T survey data. The $P_{k}$ 's in this plot are ordered 
This is the author's version of an article that has been published in this journal. Changes were made to this version by the publisher prior to publication. The final version of record is available at http://dx.doi.org/10.1109/TASC.2014.2360986

TABLE I

Global AGREEMENT QUANTIFIED By THE RMS VAlues $\epsilon_{i}$ OF $\delta V_{i}\left(P_{k}\right)$

\begin{tabular}{lllll} 
CCL5T $_{e}$ & $f(\mathrm{~Hz})$ & $\epsilon_{Z}$ & $\epsilon_{R}$ & $S F$ \\
\hline \hline$(\mathrm{x}, \mathrm{y}, \mathrm{z})$ & 9 & $\approx 0.5$ & $\approx 0.5$ & 1 \\
$(\mathrm{x}, \mathrm{y}, \mathrm{z})$ & 1 & $0.013(1)$ & $0.017(6)$ & 0.9755 \\
$(\mathrm{x}, \mathrm{y}, \mathrm{z}=\bar{z})$ & 1 & $0.014(8)$ & $0.019(4)$ & 0.9755 \\
\hline
\end{tabular}

differently from those shown in Fig. 3. Each distinct peak is the value from the coil closest to the source, at one of 52 locations. This plot demonstrates clearly that $\mathrm{z}$-axis variations larger than $1 \mathrm{~mm}$ can be detected in the least sensitive radial component of magnetic flux.

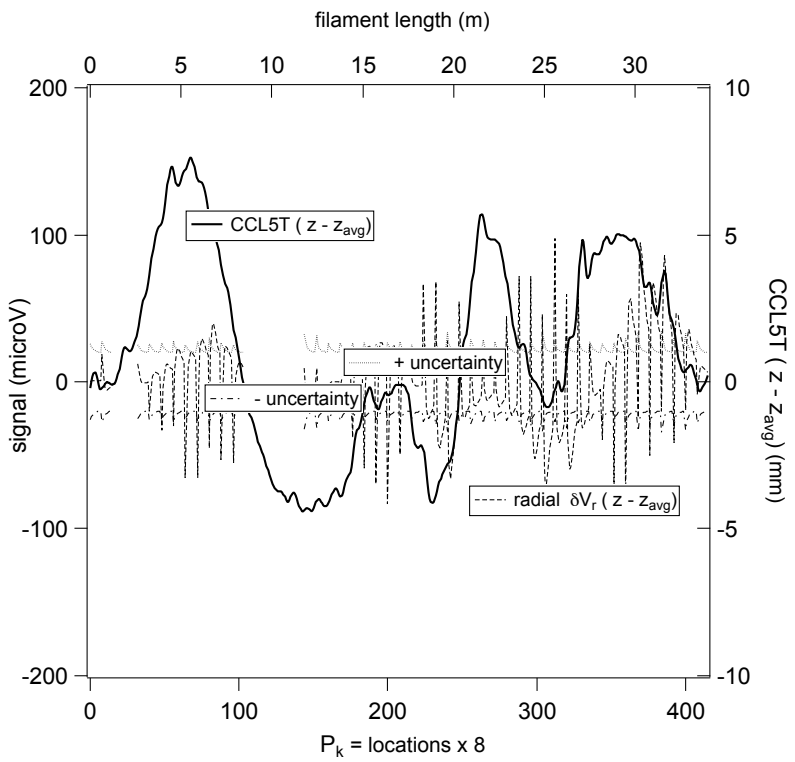

Fig. 4. Left: signal difference associated to the z-axis variations of the filament and total noise. Right: height modulation $\operatorname{CCL5T}(z-\bar{z})$

\section{INVERSE PROBLEM: PRELIMINARY RESULTS}

All data sets recorded during the survey of a winding pack will be used simultaneously to reconstruct the shape of the CCL by solving an inverse problem. We seek the CCL shape changes (compared to nominal), so that the norm of the shape change $\|\delta a\|$ as well as the norm of the difference between the measured and computed values, $\left\|\left(V_{e}-V_{m}\right)\right\|$ are minimized. A first solution of Eq. 2 in Ref. [15] was obtained using the singular value decomposition (SVD) approach [16], [20] and tested (but not fully validated) on available experimental data $V_{i, e}\left(P_{k}\right)$. Shape changes are described with Fourier series. Results along the $\mathrm{z}$ axis are shown in Fig. 5. The average $\bar{z}_{r c}$ of the reconstructed CCL obtained using CCL5T as initial conditions is $3 \mathrm{~mm}$ higher the value $\bar{z}$ obtained by survey, as if the cable is globally closer to the detectors. This is unlikely since instrumental and calibration uncertainties cannot explain such a difference. The largest $\mathrm{z}$ axis deviations introduced by the reconstruction algorithm are visible near 5, 12, 23, and 28 $m$ of the circumference. Their "wavelength" are commensurate with the type of cable and the details of the experiment, their magnitude appears too large, however. Shape changes introduced in the xy plane (not shown) indicate a small shrinkage of the entire filament shape.

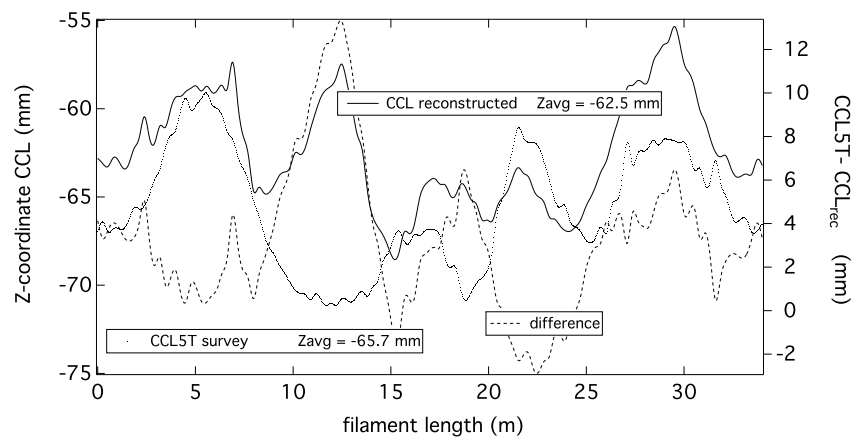

Fig. 5. Left: surveyed $\operatorname{CCL5T}(z)$ and reconstructed $\operatorname{CCL}(z)$ obtained with the inversion algorithm. Right: difference.

\section{CONCLUSION}

Using the forward approach, and a reasonably well confined 5 turns current filament lying in one (1 turn) groove of a full scale radial plate, excited by an ac current, we recorded magnetic flux maps of two orthogonal field components. The average distance between two measurement locations is 650 $\mathrm{mm}$; no spatial oversampling. Neglecting eddy currents, (i.e. using magneto-static only) the relative agreement between measured and modeled values is below $2 \%$. The sensitivity (Fig. 4) is sufficient to detect a $1 \mathrm{~mm}$ shape variations along the $\mathrm{z}$-axis. The negative impact of eddy currents manifests itself by an apparent position dependent signal amplitude loss, and decreases with decreasing frequency.

The final TF coil will have 134 turns and the distance between the detectors and the CCL must increase to $\approx 0.5$ $\mathrm{m}$, so that a reduction of the excitation frequency to $0.2 \mathrm{~Hz}$ is possible without signal amplitude loss. For the magnetic survey of this object, we are building a carbon fiber detector holder [21] to allow the acquisition of six data sets (see sketch in Fig. 6 of ref [15]). Four sets will be recorded following the perimeter of the coil along the four edges of the coil's cross section. This shall provide data from inside and outside of the D-shaped coil. Two more sets will be acquired in gradiometer configuration and deliver $V_{r}\left(z^{+}\right)$and $V_{r}\left(z^{-}\right)$data from which, using the XY symmetry of the coil, robust values of $\operatorname{CCL}(z)$ will be obtained.

At this preliminary level, the reconstructed CCL indicates shape differences larger than the experimental sensitivity obtained from Fig. 4. A robust check of the inversion procedure requires two separate experiments (see for example [14]) in which controlled shape changes are introduced, and combined with forward computations.

Further, full validation of this measurement approach will be performed with a 22 winded turns full scale radial plate, followed by a 134 winded turns TF coil.

\section{DISCLAIMER}

The views and opinions expressed herein do not necessarily reflect those of the ITER Organization. 
This is the author's version of an article that has been published in this journal. Changes were made to this version by the publisher prior to publication. The final version of record is available at http://dx.doi.org/10.1109/TASC.2014.2360986

\section{REFERENCES}

[1] J. Knaster et al. "ITER non-axisymmetric error fields induced by its magnet system", Fusion Engineering and Design vol. 86, pp. 1053-1056, 2011.

[2] N. Mitchell, A. Devred, P. Libeyre, B. Lim, F. Savary and ITER Magnet Division, "The ITER magnets: design and construction status", IEEE Trans. Appl. Supercond. vol. 22, no. 3, June 2012, Art. No. 4200809.

[3] ITER technical drawing, ID 28HP9H, drawing 000885.

[4] J. Knaster and E. Baynham, "The determination of the current center line of the TF coils of ITER", IEEE Trans. Appl. Supercond. vol. 20, no 3, pp. 1475-1478, June 2010.

[5] V. Amoskov et al. "Some aspects of winding geometry control for ITER superconducting coils using magnetic measurements" IEEE Trans. Appl. Supercond. vol. 21, no. 3, pp. 2308-2311, June 2011.

[6] L. Deniau, E. Baynham, M. Buzio, J. Knaster and F. Savary, "Magnetic measurement of the current center line of the toroidal field coil of ITER at room temperature", IEEE Trans. Appl. Supercond. vol. 22, no. 3, June 2012, Art. No 9001704.

[7] B. Bellesia et al. "Magnetic measurements for magnets manufacturing tolerances assessment", IEEE Trans. Appl. Supercond. vol. 24, no. 3, June 2014, Art. No 9000405

[8] A. Bonito Oliva, A. Formisano, J. Knaster, R. Martone, A. Portone and P. Testoni, "Magnetically based acceptance procedures for ITER toroidal coils: an assessment of performances", IEEE Trans. Appl. Supercond. vol. 22, no. 3, June 2012, Art. No 4200704

[9] A. Bonito Oliva et al."Characterization of Deformed Magnets from External Magnetic Measurements", IEEE Trans. on Magnetics vol. 50, no. 2, Feb. 2014, Art. No 7024804 .

[10] F. Savary et al. "Status report on the toroidal field coils of the ITER project", IEEE Trans. Appl. Supercond. vol. 20, no. 3, pp. 381-384, June 2010.

[11] N. Koizumi et al. "Development of the ITER toroidal field coil winding pack in Japan", IEEE Trans. Appl. Supercond. vol. 20, no. 3, pp. 385-388, June 2010.

[12] J. Knaster et al. "Final assembly and installation of the ITER TF coils", IEEE Trans. Appl. Supercond. vol. 18, no. 2, pp. 495-499, June 2008.

[13] E. Baynham, R. Gallix, J. Knaster, N. Mitchell, and F. Savary, "The insertion of the WP in the structural casing of the TF coils of ITER", IEEE Trans. Appl. Supercond. vol. 20, no. 3, pp. 389-392, June 2010.

[14] K. Buhagiar, Ph. Lerch, A. Gabard, M. Buzio, S. Sanlippo, A. Foussat, "Magnetic survey of large magnets: a 1:5 scale model system", Proc. 20th IMEKO TC4, International Symposium, pp. 917-922, September 2014, ISBN-14: 978-92-990073-2-7.

[15] Ph. Lerch et al. "ITER TF coils: CCL at room temperature", IEEE Trans. Appl. Supercond. vol. 24, no. 3, June 2014, Art No. 4201604.

[16] G. H. Golub and W. Kahan, "Calculating the singular values and pseudoinverse of a matrix", J. Soc. Indust. Appl. Math. Numer Anal. vol. B2, no. 2, pp. 205-224, 1965.

[17] 8 channels PCI-6231 ADC card, National Instrument.

[18] C. V. Dodd, "Analytical solutions to eddy-current probe-coil problems", J. Appl. Phys. vol. 39, no. 6, pp. 2829-2838, May 1968.

[19] L. Hogben, "Handbook of Linear Algebra", Chapman \& Hall/ CRC, 2007.

[20] G. H. Golub and C. Reinch, "Singular Value Decomposition and Least Squares solutions", Numerische Mathematik vol. 14, no. 5, pp. 403-420, 1970.

[21] D. Wassmer, "Development of a measurement system carrier for the ITER toroidal field coils: design and evaluation", bachelor thesis, Technical University, Konstanz, Germany, 2014 unpublished. 